\title{
Probing the spin structure of the fractional quantum Hall magnetoroton with polarized Raman scattering
}

\author{
Dung Xuan Nguyen $\odot^{1, *}$ and Dam Thanh Son ${ }^{2}$ \\ ${ }^{1}$ Brown Theoretical Physics Center and Department of Physics, Brown University, 182 Hope Street, Providence, Rhode Island 02912, USA \\ ${ }^{2}$ Kadanoff Center for Theoretical Physics, University of Chicago, 933 East 56th Street, Chicago, Illinois 60637, USA
}

(Received 14 February 2021; accepted 30 March 2021; published 13 April 2021)

\begin{abstract}
Starting from the Luttinger model for the band structure of GaAs, we derive an effective theory that describes the coupling of the fractional quantum Hall $(\mathrm{FQH})$ system with photons in resonant Raman scattering experiments. Our theory is applicable in the regime when the energy of the photons $\omega_{0}$ is close to the energy gap $E_{G}$, but $\left|\omega_{0}-E_{G}\right|$ is much larger than the energy scales of the quantum Hall problem. In the literature, it is often assumed that Raman scattering measures the dynamic structure factor $S(\omega, \mathbf{k})$ of the FQH. However, in this paper, we find that the light scattering spectra measured in the experiments are proportional to the spectral densities of a pair of operators which we identified with the spin-2 components of the kinetic part of the stress tensor. In contrast with the dynamic structure factor, these spectral densities do not vanish in the long-wavelength limit $k \rightarrow 0$. We show that Raman scattering with circularly polarized light can measure the spin of the magnetoroton excitation in the FQH system. We give an explicit expression for the kinetic stress tensor that works on any Landau level and which can be used for numerical calculations of the spectral densities that enter the Raman scattering amplitudes. We propose that Raman scattering provides a way to probe the bulk of the $v=\frac{5}{2}$ quantum Hall state to determine its nature.
\end{abstract}

DOI: 10.1103/PhysRevResearch.3.023040

\section{INTRODUCTION}

The fractional quantum Hall effect (FQHE) was discovered in experiment [1]. Fractional quantum Hall $(\mathrm{FQH})$ systems support a host of intriguing physical phenomena; they are also a playground for many exotic theoretical ideas ranging from anyons [2-4] to superconductivity [5], skyrmion [6], and bimetric gravity [7], to name a few. Anyonic excitations in nonabelian $\mathrm{FQH}$ states such as the Moore-Read state at filling fraction $\frac{5}{2}$ [8] may provide the building blocks for a topological quantum computer [9]. However, FQHE is still one of the most difficult and important unsolved problems of modern physics.

In a classic paper, Girvin et al. [10] proposed a single mode approximation for the FQHE in which the only excitation of the FQH system is a gapped charge-neutral mode called "magnetoroton." In the original treatment, the magnetoroton was interpreted as the charge density wave in the lowest Landau level (LLL). The dispersion relation of this neutral mode has a minimum at the wavelength of the order of the magnetic length $\ell_{B}$, which imitates the behavior of the roton mode in superfluid ${ }^{4} \mathrm{He}$. Experiments have confirmed the existence of the magnetoroton mode in light scattering experiments

\footnotetext{
*dung_x_nguyen@brown.edu

Published by the American Physical Society under the terms of the Creative Commons Attribution 4.0 International license. Further distribution of this work must maintain attribution to the author(s) and the published article's title, journal citation, and DOI.
}

[11-13]. ${ }^{1}$ In the widely accepted theoretical interpretation of these experiments, the light scattering intensity has been associated with the dynamical structure factor $S(\omega, \mathbf{k})$ in the LLL [15]. However, in the LLL limit, the dynamical structure factor goes to zero as $k^{4}[10]$ in the limit where the momentum of the magnetoroton $k$ goes to zero. On the other hand, the Raman signal seems to persist down to $k=0$, signaling that the identification of the intensity of Raman scattering with the dynamic structure factor may not be correct.

In this paper, we provide a theoretical treatment for Raman scattering experiments. We first note that the problem of Raman scattering involves many energy scales. The largest energy scale is that of the semiconductor gap $E_{G}$ and the photon energy $\omega_{0}$. The next scale is the distance between the Landau levels of the conduction-band electrons $\omega_{c}=e B / m^{*} c$, and the smallest energy scale is the Coulomb interacting energy between these electrons $\Delta$. We assume a hierarchy

$$
\Delta \ll \omega_{c} \ll E_{G} .
$$

Only the physics at the scale $\Delta$ is "hard," i.e., nonperturbative or strongly correlated, while the physics at the scales $E_{G}$ and $\omega_{c}$ are weakly coupled. To solve the Raman scattering problem effectively, one needs to separate out the nonperturbative, strongly correlated physics at the scale $\Delta$ from the perturbative, weak coupling physics at the other scales. (This is like the philosophy of "factorization" in quantum chromodynamics [16], where the perturbative physics at the hadronic scale

\footnotetext{
${ }^{1}$ For an alternative interpretation of the mode, see Ref. [14].
} 
is separated from the perturbative physics of higher energy scales.)

We perform this "factorization" procedure in two steps. The first is to integrate out the energy scale $E_{G}$. Starting with the Luttinger Hamiltonian for GaAs [17], we introduce a coupling between the lowest conduction band and highest valence band due to the interaction with light waves. We focus on the regime of resonant Raman scattering in which the frequency of the incoming light is close to the semiconductor energy gap $\omega_{0} \approx E_{G}$. Under the assumption that the detuning between the frequency of light and the gap $\left|\omega_{0}-E_{G}\right|$ is larger than both energy scales of the Hall effect $\omega_{c}$ and $\Delta$, we integrate out valence bands to obtain the coupling of the conduction-band electron to the photon. The second step is to do projection to a single Landau level. The result is an effective coupling of the Raman photons to operators acting on a single Landau level.

Our result differs drastically from that of Ref. [15]. We find that, instead of measuring the spectral density of the density operator (the dynamic structure factor), the Raman scattering experiment measures the spectral densities of the operators that can be called the "kinetic stress tensor" operators $T_{i j}^{\mathrm{kin}}=\frac{1}{m^{*}} \partial_{i} \psi^{\dagger} \partial_{j} \psi$. These operators are the components of the stress tensor that arise from the kinetic energy term in the many-body Hamiltonian. For simple model Hamiltonians leading to exact zero-energy trial wave functions, the kinetic stress tensor coincides with the full stress tensor, but that is not the case for the general case, including that of the Coulomb interaction. Moreover, in the LLL limit, in the longwavelength limit, the only components of the kinetic stress tensor that have nonvanishing spectral densities are the two spin- 2 components $T_{z z}^{\mathrm{kin}}$ and $T_{\bar{z}}^{\mathrm{kin}}$ (the spin- 0 component $T_{z \bar{z}}^{\mathrm{kin}}$ has vanishing spectral density in the limit $k \rightarrow 0$ ).

Recent theoretical papers [18-21] have advanced a proposal on the interpretation of the magnetoroton. According to this proposal, the magnetoroton is the quantum dynamical metric, and at the long-wavelength regime, $k \sim 0$ has an angular momentum $=2$ in the direction of the applied magnetic field [19]. To determine the spin of the magnetoroton, it has been suggested [19-21] that the spin of the magnetoroton can be detected through polarized Raman scattering.

We show in this paper how Raman scattering with circularly polarized light can indeed be used to confirm the spin of the magnetoroton, including the sign. The basic idea is very simple: if angular momentum is exactly conserved, an $\mathrm{FQH}$ state can absorb only one specific circular polarized photon to excite a spin-2 magnetoroton mode and emit a photon with opposite circular polarization. However, the real system does not have full rotational symmetry, but only $C_{4}$, so spins 2 and -2 are not distinct from the point of view of symmetry. In this paper, we carefully analyze the resonant Raman scattering using the Luttinger model of GaAs. We determine the magnitude of the effect of nonconservation of angular momentum through the Luttinger parameters and show that it is numerically small (of the order of $\frac{1}{40}$ ).

We organize the paper as follows. We start Sec. II by introducing our theoretical model for Raman scattering of an FQH state. In Sec. III, we present the calculation of the intensity of circularly polarized light scattering. We show that, in contrast with previous theoretical proposals, the peaks in scattering intensity, at the long-wavelength regime, was obtained mainly due to the poles in the correlation functions of the kinetic part of stress tensor $\left\langle T^{\mathrm{kin}} T^{\mathrm{kin}}\right\rangle$. We relate the cross-section of Raman scattering by circularly polarized light with the spectral densities of the stress tensor. In Sec. IV, we derive the explicit formulas for the stress tensor projected to a Landau level, which can be used to evaluate numerically the spectral function. We then conclude the paper in Sec. V. The Appendices are devoted to the details of the calculation.

\section{MODEL}

In GaAs, the light hole and heavy hole bands make the main contribution to the Raman scattering process [15]. Thus, the spin-off bands can be ignored in this sense. We consider the effective Lagrangian, with only the conduction band $\psi^{\alpha}$ and $j=\frac{3}{2}$ valence bands (which is nothing but light hole and heavy hole bands) $\chi_{i}^{\alpha}$. In the notation, $\alpha=1,2$ represents the spinor index, and $i=1,2,3$ represents three components of the $p$ wave function. The ("Rarita-Schwinger") constraint is imposed

$$
\left(\sigma^{i}\right)^{\alpha}{ }_{\beta} \chi_{i}^{\beta}=0,
$$

which projects out the $j=\frac{1}{2}$ part from $\chi_{i}^{\alpha}$. Consider the Luttinger Hamiltonian for the heavy hole and light hole (within approximation $\mathbf{k} \approx 0$ ) [17]

$$
\begin{aligned}
H= & \frac{1}{m}\left\{\left(\gamma_{1}+\frac{5 \gamma_{2}}{2}\right) \frac{\mathbf{D}^{2}}{2}-\gamma_{2} \sum_{i} J_{i}^{2} D_{i}^{2}-2 \gamma_{3}\left[\left\{J_{x} J_{y}\right\}\left\{D_{x} D_{y}\right\}\right.\right. \\
& \left.\left.+\left\{J_{y} J_{z}\right\}\left\{D_{y} D_{z}\right\}+\left\{J_{z} J_{x}\right\}\left\{D_{z} D_{x}\right\}\right]+\frac{e}{c} \kappa \mathbf{J} \cdot \mathbf{B}\right\},
\end{aligned}
$$

where $\gamma_{1,2,3}$ are the Luttinger parameters, $m$ is the mass of the electron, $J_{i}$ are the $S O(3)$ generators: $\left(J_{i}\right)_{j k}=-i \epsilon_{i j k}$, $D_{i} \chi=\left(\partial_{i}-i \frac{e}{c} A_{i}\right) \chi$ is the covariant derivative, $\{\cdot\}$ denotes symmetrization, e.g., $\left\{D_{x} D_{y}\right\} \equiv \frac{1}{2}\left(D_{x} D_{y}+D_{y} D_{x}\right)$, and $e<0$ is the electric charge of the electron. Using the equality

$$
\left\{J_{i} J_{j}\right\}\left\{D_{i} D_{j}\right\}=(\mathbf{J} \cdot \mathbf{D})^{2}-\frac{e}{2 c} \mathbf{J} \cdot \mathbf{B},
$$

we can rewrite the Hamiltonian in Eq. (3) as

$$
\begin{aligned}
H= & \frac{1}{m}\left[\left(\gamma_{1}+\frac{5 \gamma_{2}}{2}\right) \frac{\mathbf{D}^{2}}{2}+\left(\gamma_{3}-\gamma_{2}\right) \sum_{i} J_{i}^{2} D_{i}^{2}-\gamma_{3}(\mathbf{J} \cdot \mathbf{D})^{2}\right. \\
& \left.+\frac{e}{c}\left(\kappa+\frac{\gamma_{3}}{2}\right) \mathbf{J} \cdot \mathbf{B}\right] .
\end{aligned}
$$

The Lagrangian for the hole band is

$$
\mathcal{L}_{v}=i \chi_{i \alpha}^{\dagger} \partial_{t} \chi_{i}^{\alpha}-\chi_{i \lambda}^{\dagger} H_{i j} \chi_{j}^{\lambda}+E_{G} \chi_{i \alpha}^{\dagger} \chi_{i}^{\alpha},
$$

where $E_{G}$ is energy gap, and the covariant derivative is $D_{i} \psi \equiv$ $\left(\partial_{i}-i \frac{e}{c} A_{i}\right) \psi$. We also have the Lagrangian of the conduction band

$$
\mathcal{L}_{c}=i \psi_{\alpha}^{\dagger} \partial_{t} \psi^{\alpha}-\frac{D_{i} \psi_{\alpha}^{\dagger} D_{i} \psi^{\alpha}}{2 m^{*}},
$$

and the coupling of the valence band and conduction band through interaction with light

$$
\mathcal{L}_{i}=e\left(P^{*} \psi_{\alpha}^{\dagger} \chi_{i}^{\alpha} E_{i}+P \chi_{i \alpha}^{\dagger} \psi^{\alpha} E_{i}\right),
$$


where $P$ is the strength of the dipole transition between the conduction and valence bands (it will be related to the parameter usually denoted as $E_{p}$ in the literature), and $E_{i}$ is the electric field of electromagnetic wave.

We consider the regime of resonant Raman scattering where the photon energy is close to the gap $\left|\omega_{0}-E_{G}\right| \ll E_{G}$. Here, we chose $\omega_{0}=\frac{\omega_{L}+\omega_{S}}{2}$, with $\omega_{L}\left(\omega_{S}\right)$ as the frequency of the incoming (scattered) photon. In this case, we can write

$$
\begin{gathered}
E_{i}=\tilde{E}_{i} e^{-i \omega_{0} t}+\tilde{E}_{i}^{*} e^{i \omega_{0} t}, \\
\chi_{i}^{\alpha}=\tilde{\chi}_{i}^{\alpha} e^{i \omega_{0} t},
\end{gathered}
$$

where $\tilde{E}_{i}$ and $\tilde{\chi}$ are slowly varying fields (e.g., fields that vary with frequencies much smaller than $\omega_{0}$ ). Substituting into the action and dropping the rapidly oscillating terms, the action can be rewritten as (for notational simplicity, we also drop the tildas in $\tilde{E}_{i}$ and $\left.\tilde{\chi}\right)$

$$
\begin{aligned}
\mathcal{L}= & i \psi_{\alpha}^{\dagger} \partial_{t} \psi^{\alpha}-\frac{D_{i} \psi_{\alpha}^{\dagger} D_{i} \psi^{\alpha}}{2 m^{*}}+e\left(P^{*} \psi_{\alpha}^{\dagger} \chi_{i}^{\alpha} E_{i}+P \chi_{i \alpha}^{\dagger} \psi^{\alpha} E_{i}^{*}\right) \\
& +i \chi_{i \alpha}^{\dagger} \partial_{t} \chi_{i}^{\alpha}+\left(E_{G}-\omega_{0}\right) \chi_{i \alpha}^{\dagger} \chi_{i}^{\alpha}-\chi_{i \lambda}^{\dagger} H_{i j} \chi_{j}^{\lambda} \\
& +\lambda_{\alpha}^{\dagger}\left(\sigma^{i}\right)^{\alpha}{ }_{\beta} \chi_{i}^{\beta}+\chi_{i \alpha}^{\dagger}\left(\sigma^{i}\right)^{\alpha}{ }_{\beta} \lambda^{\beta} .
\end{aligned}
$$

The last two terms are the Lagrangian multiplier for constraints in Eq. (2).

Integrating out $\chi$ and $\lambda$ is equivalent to solving the field equations and the constraints

$$
\begin{gathered}
e P \psi^{\alpha} E_{i}^{*}+\left(E_{G}-\omega\right) \chi_{i}^{\alpha}+\left(\sigma^{i}\right)^{\alpha}{ }_{\beta} \lambda^{\beta}+i \partial_{t} \chi_{i}^{\alpha}-H_{i j} \chi_{j}^{\alpha}=0, \\
\left(\sigma^{i}\right)^{\alpha}{ }_{\beta} \chi_{i}^{\beta}=0 .
\end{gathered}
$$

We will focus on the regime where the photon energy is not too close to the gap. More precisely, we will assume that the detuning $\left|\omega_{0}-E_{G}\right|$ is still much larger than the distance between the Landau levels in the bands

$$
\omega_{c} \equiv \frac{|e| B}{m c} \ll\left|\omega_{0}-E_{G}\right| \ll \omega_{0} .
$$

In the $\mathrm{FQH}$ regime and holes, the typical momentum scale is $1 / \ell_{B}$; one has

$$
H \sim \frac{1}{m} D^{2} \sim \frac{e B}{m c} .
$$

This means that one can solve Eq. (12), ignoring the $\partial_{t}$ and $H$ terms,

$$
\begin{aligned}
\chi_{i}^{\alpha}= & -\frac{e P}{3\left(E_{G}-\omega_{0}\right)}\left[2 \psi^{\alpha} E_{i}^{*}+i \epsilon^{i j k}\left(\sigma^{j}\right)^{\alpha}{ }_{\beta} \psi^{\beta} E_{k}^{*}\right] \\
& +O\left(\frac{\omega_{c}}{\left|E_{G}-\omega_{0}\right|}\right) .
\end{aligned}
$$

Substituting this solution into the action, we then find the effective action for $\psi$ alone. In fact, since $\chi$ is the saddle point, an error of the order of $O\left(\omega_{c} /\left|E_{G}-\omega_{0}\right|\right)$ translated into an error $O\left(\omega_{c}^{2} /\left|E_{G}-\omega_{0}\right|^{2}\right)$ in the action; thus, it is justified to also keep the terms $\chi^{\dagger} \partial_{t} \chi$ and $\chi^{\dagger} H \chi$ when we do the substitution in Eq. (16).
To simplify the result, we assume that the electrons are fully polarized with $\operatorname{spin} s_{z}=\frac{1}{2}$, so

$$
\psi^{\alpha}=\left(\begin{array}{l}
\psi \\
0
\end{array}\right) .
$$

We assume the incoming and outgoing photons to have momenta along the $z$ direction, so $E_{i}$ are independent of $x$ and $y$. In this case, the Lagrangian describing the interaction of the conduction-band electron with the Raman photons has the form

$$
\mathcal{I}_{\text {int }}=V_{\alpha \beta}\left(E^{*}\right)^{\alpha} E^{\beta},
$$

where $V_{\alpha \beta}$ is some operators quadratic over $\psi$.

The photon spin then points along or opposite to the $z$ axis, corresponding to the two circular polarizations. We will distinguish processes in which the direction of the spin of the photon flips from those in which the photon spin does not change direction. Introducing the complex coordinates (in the quantum Hall convention)

$$
\begin{aligned}
z & =x-i y, \quad \bar{z}=x+i y, \quad \partial_{z}=\frac{1}{2}\left(\partial_{x}+i \partial_{y}\right), \\
\partial_{\bar{z}} & =\frac{1}{2}\left(\partial_{x}-i \partial_{y}\right),
\end{aligned}
$$

so

$$
E^{z}=E_{x}-i E_{y}, \quad E^{\bar{z}}=E_{x}+i E_{y},
$$

and

$$
D_{z}=\frac{1}{2}\left(D_{x}+i D_{y}\right), \quad D_{\bar{z}}=\frac{1}{2}\left(D_{x}-i D_{y}\right),
$$

the interaction Lagrangian can be written as

$$
\mathcal{I}_{\text {int }}=V_{z z}\left(E^{\bar{z}}\right)^{*} E^{z}+V_{\bar{z} \bar{z}}\left(E^{z}\right)^{*} E^{\bar{z}}+V_{z \bar{z}}\left(E^{\bar{z}}\right)^{*} E^{\bar{z}}+V_{\bar{z} z}\left(E^{z}\right)^{*} E^{z} .
$$

The terms responsible for scatterings with a switch in the photon helicity contain $V_{z z}$ and $V_{\bar{z} \bar{z}}$. Direct calculation yields

$$
\begin{aligned}
V_{\bar{z} \bar{z}}= & \frac{e^{2}|P|^{2}}{6\left(E_{G}-\omega_{0}\right)^{2}}\left[\left(\gamma_{3}+\gamma_{2}\right) D_{z} \psi^{\dagger} D_{z} \psi\right. \\
& \left.-\left(\gamma_{3}-\gamma_{2}\right) D_{\bar{z}} \psi^{\dagger} D_{\bar{z}} \psi\right], \\
V_{z z}= & \frac{e^{2}|P|^{2}}{6\left(E_{G}-\omega_{0}\right)^{2}}\left[\left(\gamma_{3}+\gamma_{2}\right) D_{\bar{z}} \psi^{\dagger} D_{\bar{z}} \psi\right. \\
& \left.-\left(\gamma_{3}-\gamma_{2}\right) D_{z} \psi^{\dagger} D_{z} \psi\right] .
\end{aligned}
$$

Terms proportional to $\gamma_{3}+\gamma_{2}$ in $V_{z z}$ and $V_{\bar{z} \bar{z}}$ preserve rotational symmetry, while terms proportional to $\gamma_{3}-\gamma_{2}$ break the angular momentum conservation by four.

It is convenient to introduce the "kinetic" stress tensor

$$
\begin{aligned}
T_{z z}^{\mathrm{kin}} & =\frac{1}{m^{*}} D_{z} \psi^{\dagger} D_{z} \psi, \quad T_{\bar{z} \bar{z}}^{\mathrm{kin}}=\frac{1}{m^{*}} D_{\bar{z}} \psi^{\dagger} D_{\bar{z}} \psi, \\
T_{z \bar{z}}^{\mathrm{kin}} & =\frac{1}{2 m^{*}}\left(D_{z} \psi^{\dagger} D_{\bar{z}} \psi+D_{\bar{z}} \psi^{\dagger} D_{z} \psi\right) .
\end{aligned}
$$

These components are the variation of the kinetic-energy part of the Hamiltonian over the external metric. This is different from the full stress tensor which contains also the variation of the potential energy over the metric. The effective operators 
coupled to the Raman photon (and flip the direction of its spin) are

$$
\begin{aligned}
V_{\bar{z} \bar{z}} & =\frac{e^{2}|P|^{2} m^{*}}{6\left(E_{G}-\omega_{0}\right)^{2}}\left[\left(\gamma_{3}+\gamma_{2}\right) T_{z z}-\left(\gamma_{3}-\gamma_{2}\right) T_{\bar{z} \bar{z}}\right], \\
V_{z z} & =\frac{e^{2}|P|^{2} m^{*}}{6\left(E_{G}-\omega_{0}\right)^{2}}\left[\left(\gamma_{3}+\gamma_{2}\right) T_{\bar{z} \bar{z}}-\left(\gamma_{3}-\gamma_{2}\right) T_{z z}\right] .
\end{aligned}
$$

For details of calculations, see Appendix A. For completeness, we also write down the operators that do not flip the photon spin

$$
\begin{aligned}
V_{\bar{z} z}= & \frac{e^{2}|P|^{2}}{\left(E_{G}-\omega_{0}\right)^{2}}\left\{\left[\theta^{*} \omega_{c}-\frac{\left(E_{G}-\omega_{0}\right)}{2}+\frac{\beta^{*} \omega_{c}}{4}\right] \rho\right. \\
& \left.+\left(2 \alpha^{*}-\beta^{*}+\gamma^{\prime *}\right) T_{z \bar{z}}+\frac{i}{2} \psi^{\dagger} \partial_{t} \psi\right\}, \\
V_{z \bar{z}}= & \frac{e^{2}|P|^{2}}{9\left(E_{G}-\omega_{0}\right)^{2}}\left\{\left[-\theta^{*} \omega_{c}-\frac{9\left(E_{G}-\omega_{0}\right)}{2}-\frac{\beta^{*} \omega_{c}}{4}\right] \rho\right. \\
& \left.+\left(6 \alpha^{*}-5 \beta^{*}+5 \gamma^{\prime *}\right) T_{z \bar{z}}+\frac{9 i}{2} \psi^{\dagger} \partial_{t} \psi\right\},
\end{aligned}
$$

where

$$
\begin{gathered}
\alpha^{*}=\frac{m^{*}}{2 m}\left(\gamma_{1}+\frac{5 \gamma_{2}}{2}\right), \quad \gamma^{*}=\frac{m^{*}}{m}\left(\gamma_{3}-\gamma_{2}\right), \\
\beta^{*}=\frac{m^{*}}{m} \gamma_{3}, \quad \theta^{*}=-\frac{m^{*}}{m}\left(\kappa+\frac{\gamma_{3}}{2}\right) .
\end{gathered}
$$

\section{SCATTERING OF CIRCULARLY POLARIZED LIGHT IN THE FQH REGIME}

In this section, we will calculate the Raman scattering on the FQH state. The magnitude of the effect can be characterized by the per-particle differential cross-section

$$
\frac{d \sigma_{\lambda \lambda^{\prime}}}{d \omega d \Omega}
$$

where $\omega$ is the difference between the energy of the incoming photon $\omega_{L}$ and the scattered photon $\omega_{S}: \omega=\omega_{L}-\omega_{S}$, $d \Omega$ is the infinitesimal solid angle of the scattered photon, and $\lambda$ and $\lambda^{\prime}$ are the indices denoting the polarization of the incoming and scattered photons. For simplicity, we consider the case when the incident and reflection light are directed perpendicularly to the sample. The light can pass through the sample, or as depicted in Fig. 1, be reflected from the sample. We will assume that both incident light and scattered lights have circular polarization, and $\lambda$ and $\lambda^{\prime}$ can be either + or - , depending on the projection of the proton spin on the $z$ axis. For example, for $\sigma_{++}$, the incident light is left handed (in the "classical optics" convention, see, e.g., Ref. [22]), and so the incident photons have spin pointing along the direction of their momentum, and the scattered light is right handed, as in Fig. 1(a), where we have the formula for cross-section per electron $[15,23]$

$$
\begin{aligned}
\frac{d \sigma_{++}(\omega)}{d \omega d \Omega} & =\frac{1}{N_{e}} \frac{\omega_{S}}{\omega_{L}} \omega_{S}^{2} \omega_{L}^{2} \sum_{f}\left|\left\langle f\left|V_{\bar{z} z}\right| i\right\rangle\right|^{2} \delta\left(\epsilon_{f}-\epsilon_{i}-\hbar \omega\right) \\
& \approx-\frac{1}{\bar{\rho}} \frac{\omega_{0}^{4}}{\pi} \operatorname{Im}\left\langle V_{\bar{z} z}^{\dagger} V_{\bar{z} z}\right\rangle_{\omega, \mathbf{0}},
\end{aligned}
$$

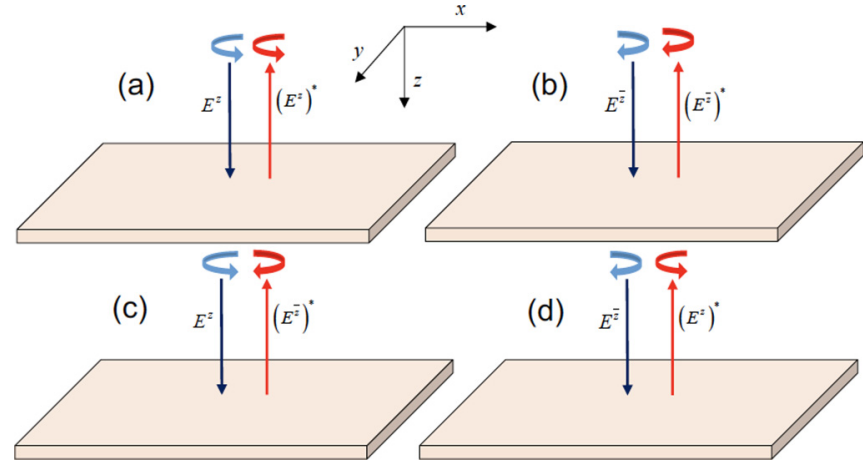

FIG. 1. Setup experiment for circular polarized light scattering.

with $N_{e}$ being the total electron number in the conductance band, $\bar{\rho}$ is the electron density in the conductance band, $\epsilon_{f}, \epsilon_{i}$ are energies of final and initial states, and

$$
\left\langle A^{\dagger} A\right\rangle_{\omega, \mathbf{k}} \equiv \int d t d \mathbf{x} e^{i \omega t-i \mathbf{k} \cdot \mathbf{x}}\left\langle T A(t, \mathbf{x}) A^{\dagger}(0, \mathbf{0})\right\rangle .
$$

Thus, we need to calculate the spectral density of the operator $V_{\bar{z} z}(\omega, 0)$.

Similarly, in the case of setups in Figs. 1(b), 1(c) and 1(d), we have

$$
\begin{aligned}
& \frac{d \sigma_{--}(\omega)}{d \omega d \Omega}=-\frac{1}{\bar{\rho}} \frac{\omega_{0}^{4}}{\pi} \operatorname{Im}\left\langle V_{z \bar{z}}^{\dagger} V_{z \bar{z}}\right\rangle, \\
& \frac{d \sigma_{+-}(\omega)}{d \omega d \Omega}=-\frac{1}{\bar{\rho}} \frac{\omega_{0}^{4}}{\pi} \operatorname{Im}\left\langle V_{z z}^{\dagger} V_{z z}\right\rangle, \\
& \frac{d \sigma_{-+}(\omega)}{d \omega d \Omega}=-\frac{1}{\bar{\rho}} \frac{\omega_{0}^{4}}{\pi} \operatorname{Im}\left\langle V_{\bar{z} z}^{\dagger} V_{\bar{z} \bar{z}}\right\rangle .
\end{aligned}
$$

The intensity of the Raman scattering in these channels is proportional to the spectral densities of the operators $V_{z \bar{z}}, V_{z z}$, and $V_{\bar{z} \bar{z}}$.

Let us now show that, in the limit of negligible Landau level mixing, the spectral densities of the operators $V_{z \bar{z}}$ and $V_{\bar{z} z}$ are zero, implying that the processes depicted in Figs. 1(a) and 1(b) do not happen. For that, we note from Eq. (28) that the integrals of $V_{z \bar{z}}$ and $V_{\bar{z} z}$ over space are linear combinations of

$$
\int d \mathbf{x} \rho, \quad \int d \mathbf{x} T_{z \bar{z}}^{\mathrm{kin}}, \quad \int d \mathbf{x} i \psi^{\dagger} \partial_{t} \psi .
$$

The first integral is the total number of particles $N_{e}$. As this quantity is conserved, it does not contribute to the spectral density. From Appendix D, we find the results for $N^{\text {th }}$ Landau level

$$
\begin{gathered}
\int d \mathbf{x} T_{z \bar{z}}^{\mathrm{kin}}=\left(N+\frac{1}{2}\right) \frac{\omega_{c}}{2} N_{e}, \\
\int d \mathbf{x} i \psi^{\dagger} \partial_{t} \psi=2 E-\left(N+\frac{1}{2}\right) \omega_{c} N_{e},
\end{gathered}
$$

where $E$ is the total energy. Both integrals reduce to conserved quantities. Thus, the Raman processes that do not involve flipping the direction of the photon spin are suppressed.

In previous experiments [11,12], the momentum transfer to the electron gas is rather small $k l_{B} \leqslant 0.15$. This implies that these experiments mainly probe the transitions where the photon spin flips sign and effectively measure the spectral 
densities of the traceless components of the kinetic stress tensor. The picture suggested here is different from the previous one suggested in Ref. [15], where the main coupling of the Raman photon to the electron liquids is through the $\psi^{\dagger} \psi A_{i}^{2}$ term in the Lagrangian. This coupling would lead to a vanishing Raman scattering at $k=0$.

Let us introduce the short-hand notation for the spectral densities of the off-diagonal components of the stress tensor

$$
\begin{aligned}
& -\operatorname{Im}\left\langle T_{z z}^{\mathrm{kin}} T_{\bar{z} \bar{z}}^{\mathrm{kin}}\right\rangle_{\omega, \mathbf{0}}=I_{+}(\omega), \\
& -\operatorname{Im}\left\langle T_{\bar{z} \bar{z}}^{\mathrm{kin}} T_{z z}^{\mathrm{kin}}\right\rangle_{\omega, \mathbf{0}}=I_{-}(\omega) .
\end{aligned}
$$

These functions should be calculated numerically. The intensity of Raman scatterings can now be expressed as

$$
\begin{aligned}
\frac{d \sigma_{+-}}{d \omega d \Omega}= & \frac{1}{\pi \bar{\rho}}\left[\frac{e^{2}|P|^{2} \omega_{0}^{2} m^{*}}{6\left(E_{0}-\omega_{0}\right)^{2}}\right]^{2}\left[\left(\gamma_{3}+\gamma_{2}\right)^{2} I_{+}(\omega)\right. \\
& \left.+\left(\gamma_{3}-\gamma_{2}\right)^{2} I_{-}(\omega)\right], \\
\frac{d \sigma_{-+}}{d \omega d \Omega}= & \frac{1}{\pi \bar{\rho}}\left[\frac{e^{2}|P|^{2} \omega_{0}^{2} m^{*}}{6\left(E_{0}-\omega_{0}\right)^{2}}\right]^{2}\left[\left(\gamma_{3}+\gamma_{2}\right)^{2} I_{-}(\omega)\right. \\
& \left.+\left(\gamma_{3}-\gamma_{2}\right)^{2} I_{+}(\omega)\right] .
\end{aligned}
$$

In Ref. [24], it was proven that $I_{+}(\omega)=0$ for the trial ground states of model Hamiltonians with contact interactions. While there is no argument that $I_{+}$should be zero for more general Hamiltonians, numerically, it was found that for Coulomb interaction $I_{+}$is much smaller than $I_{-}$for the Laughlin $v=\frac{1}{3}$ state $[20,25]$.

If one ignores $I_{+}$compared with $I_{-}$, we find the ratio of scattered light intensity of experiment setups in Figs. 1(c) and $1(\mathrm{~d})$

$$
\frac{I_{-+}(\omega=\Delta)}{I_{+-}(\omega=\Delta)}=\frac{\left(\gamma_{3}+\gamma_{2}\right)^{2}}{\left(\gamma_{3}-\gamma_{2}\right)^{2}} .
$$

The ratio only depends on the Luttinger parameters. Moreover, the fact that $I_{+-}$will vanish if $\gamma_{3}-\gamma_{2}=0$ suggests that the signal of $I_{+-}$is due to rotational symmetry breaking. These results confirm that at zero momentum $(\mathbf{k}=0)$, the magnetoroton excitation has spin 2 in the $\hat{z}$ direction. However, in the case of finite momentum, the magnetoroton excitation will be a mix of modes with spin +2 and spin -2 in the $\hat{z}$ direction, which was suggested in the previous paper [19].

The numerical values for the Luttinger parameters of GaAs are [26]

$$
\gamma_{1}=6.9, \quad \gamma_{2}=2.1, \quad \gamma_{3}=2.9, \quad \kappa=1.2 .
$$

Substituting these parameters into Eq. (44) yields the ratio of intensities

$$
\frac{I_{-+}(\omega=\Delta)}{I_{+-}(\omega=\Delta)} \approx 40 .
$$

Note that this relies on the assumption that $I_{+}=0$, which is not expected to hold exactly for the Coulomb interaction. However, if $I_{+}$is smaller than $I_{-}$, one still expects that $I_{-+} \ll I_{+-}$for $v=\frac{1}{3}$ states. This is also expected for the Jain states $v=n /(2 n+1)$, in which the composite fermion theory implies that the magnetoroton has the same sign of spin as in the $v=\frac{1}{3}$ state. In the particle-hole conjugate Jain states $v=(n+1) /(2 n+1)$, in contrast, one expects that $I_{-+} \gg$ $I_{+-}[27,28]$.

\section{STRESS TENSOR PROJECTED ON A LANDAU LEVEL}

As we have seen from the previous section, to obtain the cross-section of polarized Raman scattering, we need to calculate the spectral function of the kinetic part of the stress tensor projected on a specific Landau level (the fractionally filled one). To enable future numerical calculations of these spectral functions, we need the expressions for the operators $T_{i j}^{\text {kin }}$ after the projection to a Landau level. In this section, we will derive the explicit form of the projected kinetic stress tensor.

We summarize the result here. For a system of particles interacting through a two-body isotropic potential $V(|\mathbf{x}-\mathbf{y}|)$ on the $N$ th Landau level, the kinetic part of the stress tensor (at zero momentum) can be written as

$$
\int d \mathbf{x} T_{i j}^{\mathrm{kin}}=\int_{\mathbf{q}} \frac{q_{i} q_{j}}{q} \frac{\partial}{\partial q}\left\{e^{-x_{q}}\left[L_{N}\left(x_{q}\right)\right]^{2}\right\} V(q) \bar{\rho}(\mathbf{q}) \bar{\rho}(-\mathbf{q}),
$$

where $\int_{\mathbf{q}} \equiv \int d \mathbf{q} /(2 \pi)^{2}$,

$$
x_{q} \equiv \frac{q^{2} \ell_{B}^{2}}{2},
$$

$L_{N}(x)$ is the Laguerre polynomial, $i j$ can be either $z z$ or $\bar{z} \bar{z}$, and $\bar{\rho}(\mathbf{q})$ is the projected density operator in momentum space [10].

The interpretation of the above equation is rather simple. Recall that the projected Hamiltonian of the system is

$$
H=\int_{\mathbf{q}} e^{-x_{q}}\left[L_{N}\left(x_{q}\right)\right]^{2} V(q) \bar{\rho}(\mathbf{q}) \bar{\rho}(-\mathbf{q}),
$$

where the form factor $e^{-x_{q}}\left[L_{N}\left(x_{q}\right)\right]^{2}$ arises from the projection to the $N$ th Landau level. Polarized Raman scattering, as explained above, has the effect of changing the effective metric in the kinetic term for the electron (making the effective mass $m^{*}$ anisotropic). This makes the Landau orbit on the $N$ th Landau level anisotropic, and the effect of that is the operator $\left(q_{i} q_{j} / q\right) \partial_{q}$ acting on the form factor.

For $N=0$, Eq. (47) reads

$$
\int d \mathbf{x} T_{z z}^{\mathrm{kin}}=-\ell_{B}^{2} \int_{\mathbf{q}} q_{z}^{2} e^{-q^{2} \ell_{B}^{2} / 2} V(q) \bar{\rho}(\mathbf{q}) \bar{\rho}(-\mathbf{q}),
$$

which is exactly the operator considered in Refs. [20,25]. Thus, the spectral densities computed in Refs. [20,25] are directly related to polarized Raman scattering on FQH states on the LLL.

For the next-to-LLL $N=1$, we have

$$
\int d \mathbf{x} T_{z z}^{\mathrm{kin}}=-\ell_{B}^{2} \int_{\mathbf{q}} q_{z}^{2} e^{-x_{q}}\left(1-x_{q}\right)\left(3-x_{q}\right) V(q) \bar{\rho}(\mathbf{q}) \bar{\rho}(-\mathbf{q}) \text {. }
$$

The general expression for the kinetic stress tensor in Eq. (47) has also been found by Yang [29]. The similar spectral function for generalized pseudopotentials and quantum Hall 
bilayers was introduced in Refs. [30,31] In the rest of this section, we provide a derivation of Eq. (47).

\section{A. Preliminaries}

We use the complex coordinates in Eq. (19) and the symmetric gauge $A_{x}=-\frac{1}{2} B y, A_{y}=\frac{1}{2} B x$. In the complex coordinates,

$$
A_{z}=\frac{1}{2}\left(A_{x}+i A_{y}\right)=i \frac{B}{4} \bar{z}, \quad A_{\bar{z}}=\frac{1}{2}\left(A_{x}-i A_{y}\right)=-i \frac{B}{4} z .
$$

Then in the symmetric gauge,

$$
\begin{aligned}
& D_{z}=\partial_{z}-\frac{i e}{c} A_{z}=\partial_{z}-\frac{\bar{z}}{4}, \\
& D_{\bar{z}}=\partial_{\bar{z}}-\frac{i e}{c} A_{\bar{z}}=\partial_{\bar{z}}+\frac{z}{4} .
\end{aligned}
$$

Note that

$$
\left[D_{z}, D_{\bar{z}}\right]=-\frac{e B}{2 c}=\frac{1}{2 \ell_{B}^{2}} .
$$

The (complex) guiding center coordinates are defined as

$$
\begin{aligned}
& Z=z-2 \ell_{B}^{2} D_{\bar{z}}=\frac{z}{2}-2 \ell_{B}^{2} \partial_{\bar{z}}, \\
& \bar{Z}=\bar{z}+2 \ell_{B}^{2} D_{z}=\frac{\bar{z}}{2}+2 \ell_{B}^{2} \partial_{z},
\end{aligned}
$$

which satisfy

$$
\left[D_{z}, Z\right]=\left[D_{z}, \bar{Z}\right]=\left[D_{\bar{z}}, Z\right]=\left[D_{\bar{z}}, \bar{Z}\right]=0,
$$

and

$$
[\bar{Z}, Z]=2 \ell_{B}^{2} \text {. }
$$

We define another set of coordinates: the relative coordinates which describe the motion around the guiding center

$$
\begin{gathered}
\zeta=2 \ell_{B}^{2} D_{\bar{z}}=\frac{z}{2}+2 \ell_{B}^{2} \partial_{\bar{z}}, \\
\bar{\zeta}=-2 \ell_{B}^{2} D_{z}=\frac{\bar{z}}{2}-2 \ell_{B}^{2} \partial_{z},
\end{gathered}
$$

which commute with $Z$ and $\bar{Z}$ [Eq. (58)] and have the commutator

$$
[\bar{\zeta}, \zeta]=-2 \ell_{B}^{2}
$$

Then $z=Z+\zeta, \bar{z}=\bar{Z}+\bar{\zeta}$. We denote the two-dimensional (2D) vector whose complex coordinates are $Z$ and $\bar{Z}$ as $\mathbf{R}$, and the vector with complex coordinates $\zeta$ and $\bar{\zeta}$ as $\tilde{\mathbf{r}}$. That means $\mathbf{x}=\mathbf{R}+\tilde{\mathbf{r}}$.

One defines two sets of creation and annihilation operators. One set moves between different Landau levels

$$
a=\sqrt{2} \ell_{B} D_{\bar{z}}=\frac{\zeta}{\sqrt{2} \ell_{B}}, \quad a^{\dagger}=-\sqrt{2} \ell_{B} D_{z}=\frac{\bar{\zeta}}{\sqrt{2} \ell_{B}},
$$

and another set moves within a Landau level

$$
b=\frac{1}{\sqrt{2} \ell_{B}} \bar{Z}, \quad b^{\dagger}=\frac{1}{\sqrt{2} \ell_{B}} Z .
$$

The orbitals are obtained by the acting creation operator on the lowest state

$$
|M, m\rangle=\frac{1}{\sqrt{M ! m !}} a^{\dagger M} b^{\dagger m}|0,0\rangle,
$$

where

$$
\langle\mathbf{x} \mid 0,0\rangle \sim e^{-|z|^{2} / 4 \ell_{B}^{2}}
$$

\section{B. The kinetic stress tensor on a Landau level}

Our task is to find the expression for the kinetic part of the stress tensor in the theory where the electrons live on one Landau level. This will be done through a field-theory formalism. The action describing electrons on the $N$ th Landau level is

$$
\begin{aligned}
S= & \int d t d \mathbf{x}\left[i \psi^{\dagger} \partial_{t} \psi+\chi^{\dagger}\left(2 \ell_{B}^{2} D_{z} D_{\bar{z}} \psi+N \psi\right)\right. \\
& \left.+\left(2 \ell_{B}^{2} D_{\bar{z}} D_{z} \psi^{\dagger}+N \psi^{\dagger}\right) \chi\right] \\
& -\frac{1}{2} \int d t d \mathbf{x} d \mathbf{x}^{\prime} V\left(\mathbf{x}-\mathbf{x}^{\prime}\right) \psi^{\dagger}(\mathbf{x}) \psi^{\dagger}\left(\mathbf{x}^{\prime}\right) \psi\left(\mathbf{x}^{\prime}\right) \psi(\mathbf{x}) .
\end{aligned}
$$

The fields $\chi$ and $\chi^{\dagger}$ are simply the Lagrangian multipliers enforcing the constraint

$$
2 \ell_{B}^{2} D_{z} D_{\bar{z}} \psi+N \psi=0,
$$

which is simply the condition that $\psi$ lies on the $N$ th Landau level.

To find the stress tensor, we first rewrite the action by integration by part

$$
\begin{aligned}
S= & \int d t d \mathbf{x}\left[i \psi^{\dagger} \partial_{t} \psi-2 \ell_{B}^{2} D_{z} \chi^{\dagger} D_{\bar{z}} \psi-2 \ell_{B}^{2} D_{z} \psi^{\dagger} D_{\bar{z}} \chi\right. \\
& \left.+N\left(\chi^{\dagger} \psi+\psi^{\dagger} \chi\right)\right]-\frac{1}{2} \int d t d \mathbf{x} d \mathbf{x}^{\prime} V\left(\mathbf{x}-\mathbf{x}^{\prime}\right) \psi^{\dagger}(\mathbf{x}) \psi^{\dagger} \\
& \times\left(\mathbf{x}^{\prime}\right) \psi\left(\mathbf{x}^{\prime}\right) \psi(\mathbf{x}),
\end{aligned}
$$

then the kinetic part of the stress tensor can be calculated from Noether's theorem:

$$
T_{j}^{i}=-\frac{\partial L}{\partial\left(\partial_{i} \phi_{a}\right)} \partial_{j} \phi_{a},
$$

where one sums over all fields $\phi_{a}$, which in our case encompass $\psi, \phi^{\dagger}, \chi$, and $\chi^{\dagger}$. For the polarized Raman experiment with perpendicularly incoming and outgoing photons, with a flipping of the photon spin, one only needs the traceless part of the stress tensor, integrated over space:

$$
\begin{aligned}
& \int d \mathbf{x} T_{z z}^{\mathrm{kin}}=-\ell_{B}^{2} \int d \mathbf{x}\left(\chi^{\dagger} D_{z}^{2} \psi+D_{z}^{2} \psi^{\dagger} \chi\right), \\
& \int d \mathbf{x} T_{\bar{z} \bar{z}}^{\mathrm{kin}}=-\ell_{B}^{2} \int d \mathbf{x}\left(\chi^{\dagger} D_{\bar{z}}^{2} \psi+D_{\bar{z}}^{2} \psi^{\dagger} \chi\right) .
\end{aligned}
$$

We can expand $\chi$ as a sum over Landau levels: $\chi=\chi_{0}+$ $\chi_{1}+\chi_{2}+\cdots$. We recall that, when acting on $\psi$ and $\chi, D_{z}$ raises and $D_{\bar{z}}$ lowers the Landau level index, while when acting on $\psi^{\dagger}$ and $\chi^{\dagger}$, they switch roles. Due to the orthogonality of wave functions on different Landau levels, only the parts of $\chi$ that are on the $(N+2)$ th and (if $N \geqslant 2)(N-2)$ th Landau 
levels contribute to the integrals in Eq. (71). We then have, for $N \geqslant 2$,

$$
\begin{aligned}
& \int d \mathbf{x} T_{z \bar{z}}^{\mathrm{kin}}=-\ell_{B}^{2} \int d \mathbf{x}\left(\chi_{N+2}^{\dagger} D_{z}^{2} \psi+D_{z}^{2} \psi^{\dagger} \chi_{N-2}\right), \\
& \int d \mathbf{x} T_{\bar{z} \bar{z}}^{\mathrm{kin}}=-\ell_{B}^{2} \int d \mathbf{x}\left(\chi_{N-2}^{\dagger} D_{\bar{z}}^{2} \psi+D_{\bar{z}}^{2} \psi^{\dagger} \chi_{N+2}\right),
\end{aligned}
$$

and for $N=0$ or 1 ,

$$
\begin{aligned}
& \int d \mathbf{x} T_{z \bar{z}}^{\mathrm{kin}}=-\ell_{B}^{2} \int d \mathbf{x} \chi_{N+2}^{\dagger} D_{z}^{2} \psi, \\
& \int d \mathbf{x} T_{\bar{z} \bar{z}}^{\mathrm{kin}}=-\ell_{B}^{2} \int d \mathbf{x} D_{\bar{z}}^{2} \psi^{\dagger} \chi_{N+2} .
\end{aligned}
$$

The equation determining $\chi$ is

$$
0=\frac{\delta S}{\delta \psi^{\dagger}}=i \partial_{t} \psi+\left(2 \ell_{B}^{2} D_{z} D_{\bar{z}}+N\right) \chi+W(\mathbf{x}),
$$

where we denoted

$$
W(\mathbf{x})=-\int d \mathbf{x}^{\prime} V\left(\mathbf{x}-\mathbf{x}^{\prime}\right) \psi^{\dagger}\left(\mathbf{x}^{\prime}\right) \psi\left(\mathbf{x}^{\prime}\right) \psi(\mathbf{x}) .
$$

For $n \neq N$, Eq. (77) implies

$$
\chi_{n}=\frac{W_{n}}{n-N} .
$$

Specifically,

$$
\begin{gathered}
\chi_{N+2}=\frac{1}{2} W_{N+2}, \\
\chi_{N-2}=-\frac{1}{2} W_{N-2} \quad(N \geqslant 2),
\end{gathered}
$$

and therefore,

$$
\begin{gathered}
\int d \mathbf{x} T_{z z}^{\mathrm{kin}}=-\frac{1}{2} \ell_{B}^{2} \int d \mathbf{x}\left(W^{\dagger} D_{z}^{2} \psi-D_{z}^{2} \psi^{\dagger} W\right), \\
\int d \mathbf{x} T_{\bar{z} \bar{z}}^{\mathrm{kin}}=\frac{1}{2} \ell_{B}^{2} \int d \mathbf{x}\left(W^{\dagger} D_{\bar{z}}^{2} \psi-D_{\bar{z}}^{2} \psi^{\dagger} W\right),
\end{gathered}
$$

where we have used the orthogonality of the functions on different Landau levels to replace $W_{N+2}$ and $W_{N-2}$ by simply $W$.

Using formulas from Appendix C, we then find

$$
\begin{aligned}
\int d \mathbf{x} T_{z z}^{\mathrm{kin}}= & -\frac{1}{2} \int_{\mathbf{q}} \ell_{B}^{2} q_{z}^{2} e^{-x_{q}} L_{N}\left(x_{q}\right)\left[L_{N}^{2}\left(x_{q}\right)-L_{N-2}^{2}\left(x_{q}\right)\right] \\
& \times V(q) \bar{\rho}(\mathbf{q}) \bar{\rho}(-\mathbf{q}), \\
x_{q} \equiv & \frac{q^{2} \ell_{B}^{2}}{2}
\end{aligned}
$$

and a similar equation where $T_{z z}$ is replaced by $T_{\bar{z} \bar{z}}$ and $q_{z}$ by $q_{\bar{z}}$. Here, $L_{N}$ is the Laguerre polynomial, and $L_{N}^{2}$ is not the square of $L_{N}$ but the associated Laguerre polynomial $L_{N}^{k}$ with $k=2$, and for the uniformity of the equation, we have defined $L_{-1}^{2}=L_{-2}^{2}=0$.

These equations can be brought to an alternative form by using the following identities involving the associated Laguerre polynomials:

$$
L_{N}^{k}(x)=L_{N}^{k+1}(x)-L_{N-1}^{k+1}(x), \quad \frac{d}{d x} L_{N}^{k}(x)=-L_{N-1}^{k+1}(x) .
$$

One can show that, for $N \geqslant 2$,

$$
L_{N}^{2}(x)-L_{N-2}^{2}(x)=L_{N}(x)-2 \frac{d}{d x} L_{N}(x),
$$

while one can also check directly that

$$
\begin{aligned}
& L_{0}(x)\left[L_{0}(x)-2 L_{0}^{\prime}(x)\right]=L_{0}(x) L_{0}^{2}(x), \\
& L_{1}(x)\left[L_{1}(x)-2 L_{1}^{\prime}(x)\right]=L_{1}(x) L_{1}^{2}(x) .
\end{aligned}
$$

We then can rewrite the kinetic part of the stress tensor for a general Landau level $N$ as

$$
\begin{aligned}
\int d \mathbf{x} T_{z z}^{\mathrm{kin}}= & -\frac{\ell_{B}^{2}}{2} \sum_{\mathbf{q}} q_{z}^{2} e^{-x_{q}} L_{N}\left(x_{q}\right)\left[L_{N}\left(x_{q}\right)-2 L_{N}^{\prime}\left(x_{q}\right)\right] \\
& \times V(q) \bar{\rho}(\mathbf{q}) \bar{\rho}(-\mathbf{q}),
\end{aligned}
$$

and another equation with the replacement $T_{z z}^{\text {kin }} \rightarrow T_{\bar{z} \bar{z}}^{\text {kin }}$ and $q_{z} \rightarrow q_{\bar{z}}$. This can be further transformed to Eq. (47).

Some remarks are in order. The kinetic part of stress tensor operators in Eq. (88) for the LLL share the same form as the spin-2 operators in Ref. [20], in which the authors calculated the normalized spectral functions. One can employ the same approach to obtain the spectral density of the stress tensor for higher Landau levels. The result will provide the estimation for Raman scattering intensity of a $\mathrm{FQH}$ system at higher Landau levels in our theoretical model. In Appendix E, we give the expression for the full stress tensor operators, including the contribution from the interaction. This can be used to calculate the spectral function of the LLL stress tensor and check the sum rules derived in Ref. [19].

\section{CONCLUSIONS}

In this paper, we have derived the coupling of the electrons in a single Landau level with applied electromagnetic waves, which effectively captures the essential physics of Raman scattering on FQH systems. We show that the electron operator responsible for Raman scattering is not the density operator, but the "kinetic stress tensor," and we derive the expression of the latter after projection to a single Landau level. We then show that, in the long-wavelength regime, the light scattering intensity in Raman experiments measures the spectral function of the kinetic part of stress tensors. Our calculation explains the scattering intensity peaks at zero momentum without relying on any momentum-nonconserving processes.

In addition, we proposed experimental setups to verify the spin-2 hypothesis of magnetoroton mode in FQH systems using Raman scattering with circularly polarized light. We show that, for a magnetoroton with a well-defined sign of spin, the ratio between light scattering intensities of different configurations of circularly polarized Raman experiments only depends on Luttinger parameters, which are well known. Measuring those ratios can confirm our theoretical model and unveil the spin of the magnetoroton excitations in a $\mathrm{FQH}$ state.

Using the explicit form of the stress tensor operator derived in this paper, one can perform the numerical calculation to obtain the spectral function of the stress tensors. One can then use the numerical results to verify the LLL sum rules proposed in Ref. [19] to predict the result of Raman scattering on states on higher Landau levels. 
Raman scattering may help resolve the question about the nature of the $v=\frac{5}{2}$ state. In recent experiments [32,33], the thermal Hall conductance at the edge of the $v=\frac{5}{2}$ state was determined to be consistent with the PH-Pfaffian state [34], but not the Pfaffian [8] or the anti-Pfaffian state [35,36], seemingly contradicting the results of numerical simulations [37]. Theoretical proposals aiming to explain this discrepancy include a disorder-stabilized thermal metal phase which is adiabatically connected to the PH-Pfaffian phase [38-41] and an incomplete thermalization on the edge [42-45]. Raman scattering provides a way to probe directly the bulk of the $v=$ $\frac{5}{2}$ state. The magnetoroton in the Pfaffian (Moore-Read) state [8] must have a spin of the same sign as in the $v=\frac{1}{3}$ Lauglin state, while in the anti-Pfaffian state [35,36], it must have the opposite sign. The PH-Pfaffian state [34], in the absence of Landau-level mixing, is particle-hole symmetric; hence, the Raman scattering probabilities $I_{+-}$and $I_{-+}$must be the same. However, it is not clear how significant the effect of Landau level mixing would be in this case. In the preprint by Haldane et al. [46], the spectral functions $I_{+}, I_{-}$for $v=\frac{5}{2}$ states was calculated numerically. The results support the conclusions in our paper.

To derive the coupling of the Raman photons to FQH electron liquid, we have assume that the detuning $\left|\omega_{0}-E_{G}\right|$ is much larger than the cyclotron energy $\omega_{c}$. This allows us to perform the first step of our "factorization" procedureintegrating out the holes-without having to think about the effect of the magnetic field on the conduction-band electrons. We suspect that our final result is valid under a weaker assumption-that the detuning is larger than the energy scale of the FQHE, i.e., of the Coulomb interaction between the conduction-band electrons. A derivation of this result would need to be a one-step procedure-integrating out the valence bands and the projecting to one Landau level at the same time. We defer this to a future paper.

\section{ACKNOWLEDGMENTS}

The authors thank D. Feldman, D. Haldane, E. Rezayi, K. Yang, and J. Wang for discussion. This paper is supported in part by the U.S. DOE Grant No. DE-FG02-13ER41958, a Simons Investigator grant, and by the Simons Collaboration on Ultra-Quantum Matter, which is a grant from the Simons Foundation (651440, DTS). D.X.N. was supported by Brown Theoretical Physics Center.

\section{APPENDIX A: DETAILED DERIVATION OF RAMAN SCATTERING COUPLING}

Here, we present the detailed derivation of the coupling of the FQH system with a photon. We define the parameters

$$
\begin{gathered}
\alpha=\frac{1}{2 m}\left(\gamma_{1}+\frac{5 \gamma_{2}}{2}\right), \quad \gamma^{\prime}=\frac{1}{m}\left(\gamma_{3}-\gamma_{2}\right), \\
\beta=\frac{1}{m} \gamma_{3}, \quad \theta=-\frac{1}{m}\left(\kappa+\frac{\gamma_{3}}{2}\right) .
\end{gathered}
$$

After integrating out fields in the valence band, we derive the effective Lagrangian for the conduction band

$$
\begin{aligned}
\mathcal{L}= & i \psi_{\alpha}^{\dagger} \partial_{t} \psi^{\alpha}-\frac{D_{i} \psi_{\alpha}^{\dagger} D_{i} \psi^{\alpha}}{2 m^{*}}+i \chi_{i \alpha}^{\dagger} \partial_{t} \chi_{i}^{\alpha}-\left(E_{G}-\omega_{0}\right) \chi_{i \alpha}^{\dagger} \chi_{i}^{\alpha} \\
& -\chi_{k^{\prime} \lambda}^{\dagger}\left[\alpha \mathbf{D}^{2}+\gamma^{\prime} \sum_{i} J_{i}^{2} D_{i}^{2}-\beta(\mathbf{J} \cdot \mathbf{D})^{2}-\frac{e}{c} \theta \mathbf{J} \cdot \mathbf{B}\right]_{k^{\prime} k} \\
& \times \chi_{k}^{\lambda} .
\end{aligned}
$$

All terms which contain valence band field $\chi_{i}^{\alpha}$ can be considered as coupling of conduction band field $\psi^{\alpha}$ with the electric field through substitution of Eq. (16). We define

$$
\begin{gathered}
\mathcal{I}_{0}=i \chi_{i \lambda}^{\dagger} \partial_{t} \chi_{i}^{\lambda}, \\
\mathcal{I}_{1}=-\left(E_{G}-\omega_{0}\right) \chi_{i \alpha}^{\dagger} \chi_{i}^{\alpha}, \\
\mathcal{I}_{\alpha}=-\alpha \chi_{k \lambda}^{\dagger} \mathbf{D}^{2} \chi_{k}^{\lambda}, \\
\mathcal{I}_{\beta}=\beta \chi_{k^{\prime} \lambda}^{\dagger}\left[(\mathbf{J} \cdot \mathbf{D})^{2}\right]_{k^{\prime} k} \chi_{k}^{\lambda}, \\
\mathcal{I}_{\gamma^{\prime}}=-\gamma^{\prime} \chi_{k^{\prime} \lambda}^{\dagger} \sum_{i}\left(J_{i}^{2}\right)_{k^{\prime} k} D_{i}^{2} \chi_{k}^{\lambda}, \\
\mathcal{I}_{\theta}=\theta \frac{e}{c} \sum_{k, k^{\prime}, \lambda} \chi_{k^{\prime} \lambda}^{\dagger}\left(\mathbf{J}_{k^{\prime} k} \cdot \mathbf{B}\right) \chi_{k}^{\lambda} .
\end{gathered}
$$

Consequently, the effective Lagrangian can be rewritten as

$$
\begin{aligned}
\mathcal{L}_{\text {eff }}= & i \psi^{\dagger} \partial_{t} \psi-\frac{\left(D_{i} \psi\right)^{\dagger} D_{i} \psi}{2 m^{*}}+\mathcal{I}_{0}+\mathcal{I}_{1}+\mathcal{I}_{\alpha}+\mathcal{I}_{\beta} \\
& +\mathcal{I}_{\gamma^{\prime}}+\mathcal{I}_{\theta} .
\end{aligned}
$$

Substitution of Eq. (16) for $\chi_{i}^{\alpha}$ in Eq. (A5) yields

$$
\mathcal{I}_{1}=-\frac{e^{2}|P|^{2}}{3\left(E_{G}-\omega_{0}\right)}\left(2 \psi^{\dagger} \psi|E|^{2}+i \epsilon^{i j k} \psi^{\dagger} \sigma^{j} \psi E_{k}^{*} E_{i}\right) \text {. }
$$

The first term in $\mathcal{I}_{1}$ is the interaction of light with charge density; the second term is the interaction of light with spin density. Considering that the electrons in the conduction band, under strong magnetic field in the $\hat{z}$ direction, only have the spin component $s_{z}=\frac{1}{2}$, we can rewrite

$$
\mathcal{I}_{1}=-\frac{e^{2}|P|^{2}}{3\left(E_{G}-\omega_{0}\right)}\left[2 E_{i} E_{i}^{*}+i\left(E_{2}^{*} E_{1}-E_{1}^{*} E_{2}\right)\right] \rho,
$$

where $\rho=\psi^{\dagger} \psi$. Since $E_{i}^{*}$ is a slow varying field under the redefinition in Eq. (9), the term with $\partial_{t} E_{i}^{*}$ in $\mathcal{I}_{0}$ is smaller than $\mathcal{I}_{1}$. We then have

$$
\mathcal{I}_{0}=i \frac{e^{2}|P|^{2}}{3\left(E_{G}-\omega_{0}\right)^{2}}\left[2 E_{i} E_{i}^{*}+i\left(E_{2}^{*} E_{1}-E_{1}^{*} E_{2}\right)\right] \psi^{\dagger} \partial_{t} \psi .
$$

To understand the next interaction terms (the Luttinger terms), we recall the formula for the kinetic part of the stress energy tensor

$$
T_{i j}^{\mathrm{kin}}=\frac{\left(D_{i} \psi\right)^{\dagger} D_{j} \psi}{2 m^{*}}+\frac{\left(D_{j} \psi\right)^{\dagger} D_{i} \psi}{2 m^{*}} .
$$

Under above assumption of spin state of electrons in the conduction band and $k_{3}=0$ (we consider a $2 \mathrm{D}$ system in the 
$x y$ plane, and the applied magnetic field is in the $\hat{z}$ direction), we can rewrite the Luttinger terms as

$$
\begin{gathered}
\mathcal{I}_{\alpha}=\frac{\alpha^{*} e^{2}|P|^{2}}{3\left(E_{G}-\omega_{0}\right)^{2}}\left[2 E_{i} E_{i}^{*}+i\left(E_{2}^{*} E_{1}-E_{1}^{*} E_{2}\right)\right]\left(T_{11}^{\mathrm{kin}}+T_{22}^{\mathrm{kin}}\right), \\
\mathcal{I}_{\beta}=-\frac{\beta^{*} e^{2}|P|^{2}}{9\left(E_{G}-\omega_{0}\right)^{2}}\left\{T_{11}^{\mathrm{kin}}\left[5 E_{2} E_{2}^{*}+5 E_{3} E_{3}^{*}+2 E_{1} E_{1}^{*}+i\left(E_{2} E_{1}^{*}-E_{1} E_{2}^{*}\right)\right]\right. \\
+T_{22}^{\mathrm{kin}}\left[5 E_{1} E_{1}^{*}+5 E_{3} E_{3}^{*}+2 E_{2} E_{2}^{*}+i\left(E_{2} E_{1}^{*}-E_{1} E_{2}^{*}\right)\right] \\
\left.-3 T_{12}^{\mathrm{kin}}\left(E_{1} E_{2}^{*}+E_{2} E_{1}^{*}\right)+i \frac{\omega_{c}}{2 c}\left[5\left(E_{2} E_{1}^{*}-E_{1} E_{2}^{*}\right)-4 i E_{a} E_{a}^{*}-2 i E_{3} E_{3}^{*}\right] \rho\right\}, \\
\mathcal{I}_{\gamma^{\prime}}=\frac{\gamma^{\prime *} e^{2}|P|^{2}}{9\left(E_{G}-\omega_{0}\right)^{2}}\left\{T_{11}^{\mathrm{kin}}\left[5 E_{2} E_{2}^{*}+5 E_{3} E_{3}^{*}+2 E_{1} E_{1}^{*}+i\left(E_{2} E_{1}^{*}-E_{1} E_{2}^{*}\right)\right]+T_{22}^{\mathrm{kin}}\left[5 E_{1} E_{1}^{*}+5 E_{3} E_{3}^{*}+2 E_{2} E_{2}^{*}+i\left(E_{2} E_{1}^{*}-E_{1} E_{2}^{*}\right)\right]\right\} \\
(\mathrm{A} 16) \\
\mathcal{I}_{\theta}=i \theta^{*} \omega_{c} \frac{e^{2}|P|^{2}}{9\left(E_{G}-\omega_{0}\right)^{2}}\left[5\left(E_{1} E_{2}^{*}-E_{2} E_{1}^{*}\right)+4 i E_{a} E_{a}^{*}+2 i E_{3} E_{3}^{*}\right] \rho,
\end{gathered}
$$

where we have defined the parameters

$$
\begin{aligned}
& \beta^{*}=\beta m^{*}, \quad \alpha^{*}=\alpha m^{*}, \\
& \gamma^{\prime *}=\gamma^{\prime} m^{*}, \quad \theta^{*}=\theta m^{*},
\end{aligned}
$$

and the cyclotron frequency

$$
\omega_{c}=-\frac{e B}{c m^{*}}=\frac{|e B|}{c m^{*}} .
$$

The effective Lagrangian for the conduction band includes the coupling of the electric field $E_{i}$ with charge density $\rho$ and the kinetic part of stress energy tensor $T_{i j}^{\mathrm{kin}}$. We have the effective interaction of the conduction band with light through $\mathcal{I}_{0}, \mathcal{I}_{1}, \mathcal{I}_{\alpha}, \mathcal{I}_{\beta}, \mathcal{I}_{\gamma^{\prime}}$, and $\mathcal{I}_{\theta}$. We can rewrite the interaction term in the convenient form for the circular polarized light scattering experiment setup in Fig. 1 . In this case, we can consider $E_{3}=0$ and $E_{3}^{*}=0$. Going to the complex coordinates in Eqs. (19) and (20), in which

$$
T_{z z}^{\mathrm{kin}}=\frac{1}{4}\left(T_{x x}^{\mathrm{kin}}-T_{y y}^{\mathrm{kin}}+2 i T_{x y}^{\mathrm{kin}}\right), \quad T_{z \bar{z}}^{\mathrm{kin}}=\frac{1}{4}\left(T_{x x}^{\mathrm{kin}}-T_{y y}^{\mathrm{kin}}-2 i T_{x y}^{\mathrm{kin}}\right), \quad T_{z \bar{z}}^{\mathrm{kin}}=\frac{1}{4}\left(T_{x x}^{\mathrm{kin}}+T_{y y}^{\mathrm{kin}}\right),
$$

we can rewrite the interaction terms as

$$
\begin{gathered}
\mathcal{I}_{0}=i \frac{e^{2}|P|^{2}}{6\left(E_{G}-\omega_{0}\right)^{2}}\left[3 E^{\bar{z}}\left(E^{\bar{z}}\right)^{*}+E^{z}\left(E^{z}\right)^{*}\right] \psi^{\dagger} \partial_{t} \psi, \\
\mathcal{I}_{1}=-\frac{e^{2}|P|^{2}}{6\left(E_{G}-\omega_{0}\right)}\left[3 E^{\bar{z}}\left(E^{\bar{z}}\right)^{*}+E^{z}\left(E^{z}\right)^{*}\right] \rho, \\
\mathcal{I}_{\alpha}=\alpha^{*} \frac{e^{2}|P|^{2}}{3\left(E_{G}-\omega_{0}\right)^{2}}\left[6 E^{\bar{z}}\left(E^{\bar{z}}\right)^{*}+2 E^{z}\left(E^{z}\right)^{*}\right] T_{z \bar{z}}^{\mathrm{kin}}, \\
\mathcal{I}_{\beta}=-\frac{\beta^{*} e^{2}|P|^{2}}{9\left(E_{G}-\omega_{0}\right)^{2}}\left\{-3 E^{z}\left(E^{\bar{z}}\right)^{*} T_{z z}^{\mathrm{kin}}-3 E^{\bar{z}}\left(E^{z}\right)^{*} T_{\bar{z} \bar{z}}^{\mathrm{kin}}+\left[5 E^{z}\left(E^{z}\right)^{*}+9 E^{\bar{z}}\left(E^{\bar{z}}\right)^{*}\right] T_{z \bar{z}}^{\mathrm{kin}}+\frac{\omega_{c}}{4}\left[E^{z}\left(E^{z}\right)^{*}-9 E^{\bar{z}}\left(E^{\bar{z}}\right)^{*}\right] \rho\right\}, \\
\mathcal{I}_{\gamma^{\prime}}=\frac{\gamma^{*} e^{2}|P|^{2}}{9\left(E_{G}-\omega_{0}\right)^{2}}\left\{-\frac{3}{2}\left[E^{z}\left(E^{\bar{z}}\right)^{*}+E^{\bar{z}}\left(E^{z}\right)^{*}\right]\left(T_{z z}^{\mathrm{kin}}+T_{\bar{z} \bar{z}}^{\mathrm{kin}}\right)+\left[5 E^{z}\left(E^{z}\right)^{*}+9 E^{\bar{z}}\left(E^{\bar{z}}\right)^{*}\right] T_{z \bar{z}}^{\mathrm{kin}}\right\}, \\
\mathcal{I}_{\theta}=\theta^{*} \frac{\omega_{c} e^{2}|P|^{2}}{18\left(E_{G}-\omega_{0}\right)^{2}}\left[E^{z}\left(E^{z}\right)^{*}-9 E^{\bar{z}}\left(E^{\bar{z}}\right)^{*}\right] \rho .
\end{gathered}
$$

We can easily check that only $\mathcal{I}_{\gamma^{\prime}}$ violates rotational invariance.

\section{APPENDIX B: THE DIPOLE-TRANSITION COEFFICIENT $P$}

Here, we follow Ref. [47] to derive an expression for the dipole-transition coefficient $P$ through the electron Bloch wave functions. The first term of Eq. (8) absorbs a photon and creates a hole in the valence band and adds an electron to the conductance band. We can rewrite this term in the Hamiltonian as

$$
-\int d x e P_{i j}^{*} \psi^{\alpha \dagger} \chi_{\alpha}^{i} E^{j}
$$


with $P_{i j}^{*}=P^{*} \delta_{i j}$. Comparing with eq. (11.23) in Ref. [47], we see that ${ }^{2}$

$$
-e P_{i j}^{*} i \omega_{p h}\left\langle n_{p h}-1\left|A^{j}\right| n_{p h}\right\rangle=-\frac{e}{m}\left\langle\psi_{\mathbf{k}}^{\alpha}, n_{p h}-1\left|A^{j} p_{j}\right| \chi_{i \alpha, \mathbf{k}}, n_{p h}\right\rangle,
$$

with $m$ being the free electron mass, and $p_{j}$ is the free electron momentum operator. Then we have

$$
P_{i j}^{*}=-\frac{i}{m \omega_{p h}}\left\langle\psi_{\mathbf{k}}^{\alpha}\left|p_{j}\right| \chi_{i \alpha, \mathbf{k}}\right\rangle,
$$

where $\psi_{\mathbf{k}}^{\alpha}$ is the Bloch wave function of the electron in the conductance band ( $s$ band)

$$
\psi_{\mathbf{k}}^{\alpha}=\frac{1}{\sqrt{N_{\text {site }}}} \sum_{a} e^{i \mathbf{k} \cdot \mathbf{x}} u_{c}^{\alpha}\left(\mathbf{x}-\mathbf{R}_{a}\right),
$$

and $\chi_{i \alpha, \mathbf{k}}$ is the Bloch wave function of the electron in the valence bands ( $p$ band)

$$
\chi_{i \alpha, \mathbf{k}}=\frac{1}{\sqrt{N_{\text {site }}}} \sum_{a} e^{i \mathbf{k} \cdot \mathbf{x}} u_{v}^{i \alpha}\left(\mathbf{x}-\mathbf{R}_{a}\right) .
$$

We have

$$
\begin{aligned}
\left\langle\psi_{\mathbf{k}}^{\alpha}\left|p_{j}\right| \chi_{i \alpha, \mathbf{k}}\right\rangle= & \frac{1}{N_{\text {site }}} \sum_{a, b} \int d^{3} \mathbf{x}\left[k_{j}\left(u^{\alpha}\right)_{c}^{*}\left(\mathbf{x}-\mathbf{R}_{a}\right) u_{v}^{i \alpha}\left(\mathbf{x}-\mathbf{R}_{b}\right)\right. \\
& \left.-i\left(u^{\alpha}\right)_{c}^{*}\left(\mathbf{x}-\mathbf{R}_{a}\right) \partial_{j} u_{v}^{i \alpha}\left(\mathbf{x}-\mathbf{R}_{b}\right)\right],
\end{aligned}
$$

The first term vanishes due to the orthogonality of the linear combination of atomic orbitals. The second term can be written as

$$
\begin{aligned}
& \left\langle\psi_{\mathbf{k}}^{\alpha}\left|p_{j}\right| \chi_{i \alpha, \mathbf{k}}\right\rangle \\
& \quad=-i \sum_{a, b} \int_{\text {unit cell }} d^{3} \mathbf{x}\left(u^{\alpha}\right)_{c}^{*}\left(\mathbf{x}-\mathbf{R}_{a}\right) \partial_{j} u_{v}^{i \alpha}\left(\mathbf{x}-\mathbf{R}_{b}\right) .
\end{aligned}
$$

Due to the angular momentum conservation, we have

$$
\begin{aligned}
& \left\langle\psi_{\mathbf{k}}^{\alpha}\left|p_{j}\right| \chi_{i \alpha, \mathbf{k}}\right\rangle \\
& \quad=-i \delta_{i j} \sum_{a, b} \int_{\text {unit cell }} d^{3} \mathbf{x}\left(u^{\alpha}\right)_{c}^{*}\left(\mathbf{x}-\mathbf{R}_{a}\right) \partial_{k} u_{v}^{k \alpha}\left(\mathbf{x}-\mathbf{R}_{b}\right) .
\end{aligned}
$$

Following Ref. [47], we obtain

$$
\left\langle\psi_{\mathbf{k}}^{\alpha}\left|p_{i}\right| \chi_{i \alpha, \mathbf{k}}\right\rangle=\left|\hat{e} \cdot \vec{p}_{\mathrm{cv}}\right|,
$$

where $\hat{e}$ is any unit vector, and $p_{\mathrm{cv}}^{x}=p_{\mathrm{cv}}^{y}=p_{\mathrm{cv}}^{z}$ with the definition

$$
p_{\mathrm{cv}}^{i}=-i \sum_{a, b} \int_{\text {unit cell }} d^{3} \mathbf{x}\left(u^{\alpha}\right)_{c}^{*}\left(\mathbf{x}-\mathbf{R}_{a}\right) \partial_{k} u_{v}^{k \alpha}\left(\mathbf{x}-\mathbf{R}_{b}\right) .
$$

We also have the relation between $\left|\hat{e} \cdot \vec{p}_{\mathrm{cv}}\right|$ and the parameter $E_{p}$ often used in the photonics literature [47]

$$
\left|\hat{e} \cdot \vec{p}_{\mathrm{cv}}\right|^{2}=\frac{E_{p} m}{2} .
$$

\footnotetext{
${ }^{2}$ We use the Coulomb gauge $A_{0}=0$ here.
}

We then obtain

$$
P^{*}=-\frac{i}{m \omega_{p h}} \sqrt{\frac{E_{p} m}{2}} .
$$

Note that the coupling depends on the frequency of photon. However, if we rewrite the coupling with electric field to coupling with gauge potential, there will be no dependence on the photon frequency. We can rewrite the coupling with photon in Eq. (8) as

$$
\mathcal{L}_{i}=e \sqrt{\frac{E_{p}}{2 m}}\left(\psi_{\alpha}^{\dagger} \chi_{i}^{\alpha} A_{i}+\chi_{i \alpha}^{\dagger} \psi^{\alpha} A_{i}^{*}\right)
$$

\section{APPENDIX C: HOW TO PROJECT TO A LANDAU LEVEL}

Here, we provide the detailed calculation for the kinetic part of the stress tensor in a specific Landau level. The electron field can be expanded in orbitals

$$
\psi(\mathbf{x})=\sum_{M m}\langle\mathbf{x} \mid M m\rangle c_{M m},
$$

where $M$ labels the Landau level, and $m$ labels the states within the Landau level. To obtain the kinetic part of the stress tensor, we need to compute

$$
\begin{aligned}
& \int d \mathbf{x} W^{\dagger}(\mathbf{x}) D_{z}^{2} \psi(\mathbf{x}) \\
& =-\int d \mathbf{x} d \mathbf{x}^{\prime} \psi^{\dagger}(\mathbf{x}) \psi^{\dagger}\left(\mathbf{x}^{\prime}\right) V\left(\mathbf{x}-\mathbf{x}^{\prime}\right) \psi\left(\mathbf{x}^{\prime}\right) D_{z}^{2} \psi(\mathbf{x}) .
\end{aligned}
$$

Inserting the expansion over modes, and limiting to the $N$ th Landau level, this becomes

$$
\begin{aligned}
& -\int d \mathbf{x} d \mathbf{x}^{\prime} \sum_{m n m^{\prime} n^{\prime}}\langle N m \mid \mathbf{x}\rangle\left\langle N m^{\prime} \mid \mathbf{x}^{\prime}\right\rangle V\left(\mathbf{x}-\mathbf{x}^{\prime}\right)\left\langle\mathbf{x}^{\prime} \mid N n^{\prime}\right\rangle D_{z}^{2} \\
& \quad \times\langle\mathbf{x} \mid N n\rangle c_{N m}^{\dagger} c_{N m^{\prime}}^{\dagger} c_{N n^{\prime}} c_{N n} .
\end{aligned}
$$

Introducing the Fourier transform of the potential

$$
V\left(\mathbf{x}-\mathbf{x}^{\prime}\right)=\int \frac{d \mathbf{q}}{(2 \pi)^{2}} e^{i \mathbf{q} \cdot\left(\mathbf{x}-\mathbf{x}^{\prime}\right)} V(\mathbf{q}),
$$

the expression becomes

$$
\begin{aligned}
& -\sum_{m n m^{\prime} n^{\prime}} \int_{\mathbf{q}} V(\mathbf{q}) \int d \mathbf{x}\langle N m \mid \mathbf{x}\rangle e^{i \mathbf{q} \cdot \mathbf{x}} D_{z}^{2}\langle\mathbf{x} \mid N n\rangle \int d \mathbf{x}^{\prime}\left\langle N m^{\prime} \mid \mathbf{x}^{\prime}\right\rangle \\
& \quad \times e^{-i \mathbf{q} \cdot \mathbf{x}^{\prime}}\left\langle\mathbf{x}^{\prime} \mid N n^{\prime}\right\rangle c_{m}^{\dagger} c_{m^{\prime}}^{\dagger} c_{n^{\prime}} c_{n} .
\end{aligned}
$$

Now we have

$$
\begin{aligned}
\int d \mathbf{x}^{\prime}\left\langle N m^{\prime} \mid \mathbf{x}^{\prime}\right\rangle e^{-i \mathbf{q} \cdot \mathbf{x}^{\prime}}\left\langle\mathbf{x}^{\prime} \mid N n^{\prime}\right\rangle & =\left\langle N m^{\prime}\left|e^{-i \mathbf{q} \cdot \hat{\mathbf{x}}}\right| N n^{\prime}\right\rangle \\
& =\left\langle N\left|e^{-i \mathbf{q} \cdot \tilde{\mathbf{r}}}\right| N\right\rangle\left\langle m^{\prime}\left|e^{-i \mathbf{q} \cdot \mathbf{R}}\right| n^{\prime}\right\rangle,
\end{aligned}
$$

but

$$
\begin{aligned}
& \left\langle N\left|e^{-i \mathbf{q} \cdot \tilde{\mathbf{r}}}\right| N\right\rangle \\
& \quad=\left\langle N\left|\exp \left[-\frac{i \ell_{B}}{\sqrt{2}}\left(q^{z} a^{\dagger}+q^{\bar{z}} a\right)\right]\right| N\right\rangle
\end{aligned}
$$




$$
\begin{aligned}
& =e^{-x_{q} / 2}\left\langle N\left|\exp \left(-\frac{i \ell_{B}}{\sqrt{2}} q^{z} a^{\dagger}\right) \exp \left(-\frac{i \ell_{B}}{\sqrt{2}} q^{\bar{z}} a\right)\right| N\right\rangle \\
& =e^{-x_{q} / 2} L_{N}\left(x_{q}\right), \quad x_{q}=\frac{q^{2} \ell_{B}^{2}}{2}
\end{aligned}
$$

therefore,

$$
\sum_{m^{\prime} n^{\prime}} \int d \mathbf{x}^{\prime}\left\langle N m^{\prime} \mid \mathbf{x}^{\prime}\right\rangle e^{-i \mathbf{q} \cdot \mathbf{x}^{\prime}}\left\langle\mathbf{x}^{\prime} \mid N n^{\prime}\right\rangle c_{m^{\prime}}^{\dagger} c_{n^{\prime}}=e^{-x_{q} / 2} L_{N}\left(x_{q}\right) \bar{\rho}(\mathbf{q}) .
$$

Analogously,

$$
\begin{aligned}
\int d \mathbf{x}\langle N m \mid \mathbf{x}\rangle e^{i \mathbf{q} \cdot \mathbf{x}} D_{z}^{2}\left\langle\mathbf{x} \mid N n^{\prime}\right\rangle & =\frac{1}{2}\left\langle N m\left|e^{i \mathbf{q} \cdot \hat{\mathbf{x}}} \frac{a^{\dagger 2}}{\ell_{B}^{2}}\right| N n\right\rangle \\
& =\frac{1}{2 \ell_{B}^{2}} \sqrt{(N+1)(N+2)}\left\langle N\left|e^{i \mathbf{q} \cdot \tilde{\mathbf{r}}}\right| N+2\right\rangle\left\langle m\left|e^{i \mathbf{q} \cdot \mathbf{R}}\right| n\right\rangle=-q_{z}^{2} e^{-x_{q} / 2} L_{N}^{2}\left(x_{q}\right)\left\langle m\left|e^{i \mathbf{q} \cdot \mathbf{R}}\right| n\right\rangle ;
\end{aligned}
$$

therefore,

$$
\sum_{m n} \int d \mathbf{x}\langle N m \mid \mathbf{x}\rangle e^{-i \mathbf{q} \cdot \mathbf{x}} D_{z}^{2}\langle\mathbf{x} \mid N n\rangle c_{m}^{\dagger} c_{n}=-q_{z}^{2} e^{-x_{q} / 2} L_{N}^{2}\left(x_{q}\right) \bar{\rho}(-\mathbf{q}) .
$$

Finally, we obtain

$$
\int d \mathbf{x} W^{\dagger}(\mathbf{x}) D_{z}^{2} \psi(\mathbf{x})=\int \frac{d \mathbf{q}}{(2 \pi)^{2}} q_{z}^{2} e^{-x_{q}} L_{N}\left(x_{q}\right) L_{N}^{2}\left(x_{q}\right) V(q) \bar{\rho}(\mathbf{q}) \bar{\rho}(-\mathbf{q}) .
$$

Similarly, for $N \geqslant 2$,

$$
\int d \mathbf{x} W^{\dagger}(\mathbf{x}) D_{\bar{z}}^{2} \psi(\mathbf{x})=\int \frac{d \mathbf{q}}{(2 \pi)^{2}} q_{\bar{z}}^{2} e^{-x_{q}} L_{N}\left(x_{q}\right) L_{N-2}^{2}\left(x_{q}\right) V(q) \bar{\rho}(\mathbf{q}) \bar{\rho}(-\mathbf{q}),
$$

while for $N=0$ or 1 , the expression is obviously zero due to the presence of two lowering operators $D_{\bar{z}}$ acting on $\psi$. Equations (C11) and (C12) are used in Sec. IV to obtain the explicit form of the kinetic part of the stress tensor on a specific Landau level.

\section{APPENDIX D: $i \psi^{\dagger} \partial_{t} \psi$ AND $T_{z \bar{z}}^{\mathrm{kin}}$}

Here, we derive the explicit form of $i \psi^{\dagger} \partial_{t} \psi$ in the LLL. The field equation reads

$$
\begin{aligned}
i \partial_{t} \psi(\mathbf{x})= & -\frac{1}{m^{*}}\left(D_{z} D_{\bar{z}}+D_{\bar{z}} D_{z}\right) \psi(\mathbf{x}) \\
& +\int d \mathbf{x}^{\prime} V\left(\mathbf{x}-\mathbf{x}^{\prime}\right) \psi^{\dagger}\left(\mathbf{x}^{\prime}\right) \psi\left(\mathbf{x}^{\prime}\right) \psi(\mathbf{x}) .
\end{aligned}
$$

We then use the constraint equation $2 \ell_{B}^{2} D_{z} D_{\bar{z}} \psi=-N \psi$ and the commutator in Eq. (55). From that, we get

$$
i \int d \mathbf{x} \psi^{\dagger} \partial_{t} \psi=I_{0}+\left(N+\frac{1}{2}\right) \omega_{c} N_{e},
$$

with $N_{e}$ being the total electron number in the conductance band and

$$
\begin{aligned}
I_{0} & =\int d \mathbf{x} d \mathbf{x}^{\prime} V\left(\mathbf{x}-\mathbf{x}^{\prime}\right) \psi^{\dagger}(\mathbf{x}) \psi^{\dagger}\left(\mathbf{x}^{\prime}\right) \psi\left(\mathbf{x}^{\prime}\right) \psi(\mathbf{x}) \\
& =\int_{\mathbf{q}} V(\mathbf{q}) e^{-q^{2} \ell_{B}^{2} / 2} \bar{\rho}(\mathbf{q}) \bar{\rho}(-\mathbf{q}) .
\end{aligned}
$$

The first term on the right-hand side of Eq. (D2) is twice the interacting energy, and the second term is the kinetic energy of electrons. ${ }^{3}$ We also have

$$
\begin{aligned}
\int d \mathbf{x} T_{z \bar{z}}^{\mathrm{kin}} & =\int d \mathbf{x} \frac{1}{2 m^{*}}\left(D_{z} \psi^{\dagger} D_{\bar{z}} \psi+D_{\bar{z}} \psi^{\dagger} D_{z} \psi\right) \\
& =\int d \mathbf{x} \frac{1}{2 m^{*}}\left(-2 \psi^{\dagger} D_{z} D_{\bar{z}} \psi-\frac{e B}{2 c} \psi^{\dagger} \psi\right) \\
& =\frac{\omega_{c}}{2}\left(N+\frac{1}{2}\right) \int d \mathbf{x} \rho \\
& =\left(N+\frac{1}{2}\right) \frac{N_{e} \omega_{c}}{2},
\end{aligned}
$$

where we used the constraint $2 \ell_{B}^{2} D_{z} D_{\bar{z}} \psi=-N \psi$ to obtain the last equality.

\section{APPENDIX E: TWO SUM RULES}

This Appendix is not directly related to Raman scattering but contains some exact sum rules. First, we write down formulas for the full stress tensor. For the model Hamiltonian, at the long-wavelength regime $\mathbf{k} \sim 0$, the full stress tensor is the same as $T^{\mathrm{kin}}$ [24]. For the general case, one needs to consider the potential-energy term in the Lagrangian. When the metric varies with time (but remains uniform in space), the potential changes according to

$$
V(\mathbf{x}) \rightarrow V\left(\sqrt{g_{i j} x^{i} x^{j}}\right),
$$

\footnotetext{
${ }^{3}$ In Ref. [24], we eliminate the second term in the LLL case by introducing the Landé factor $\mathfrak{g}=2$.
} 
and so the Fourier transform changes as

$$
V(\mathbf{q}) \rightarrow V\left(\sqrt{g^{i j} q_{i} q_{j}}\right) .
$$

Since the stress tensor is given by $\delta S=\frac{1}{2} \int d x T_{i j} \delta g_{i j}$, the potential part of the stress-energy tensor is then

$$
\int d \mathbf{x} T_{i j}^{\mathrm{pot}}=\frac{1}{2} \int_{\mathbf{q}} \frac{q_{i} q_{j}}{q} V^{\prime}(q) \rho(\mathbf{q}) \rho(-\mathbf{q}) .
$$

This can be projected to the $N$ th Landau level to become

$$
\int d \mathbf{x} T_{i j}^{\mathrm{pot}}=\frac{1}{2} \int_{\mathbf{q}} e^{-x_{q}}\left[L_{N}\left(x_{q}\right)\right]^{2} \frac{q_{i} q_{j}}{q} V^{\prime}(q) \bar{\rho}(\mathbf{q}) \bar{\rho}(-\mathbf{q}) .
$$

It is interesting to compare the formula with that of the kinetic stress tensor, Eq. (47): the "stretching operator" $\left(q_{i} q_{j} / q\right) \partial_{q}$ now acts on the potential $V(q)$ instead of acting on the form factor. The full stress tensor is the sum of the kinetic stress tensor, Eq. (88), and the potential stress tensor, Eq. (E4). It can be written as

$$
\int d \mathbf{x} T_{i j}^{\text {full }}=\frac{1}{2} \sum_{\mathbf{q}} \frac{q_{i} q_{j}}{q} \frac{\partial}{\partial q}\left\{e^{-x_{q}}\left[L_{N}\left(x_{q}\right)\right]^{2} V(q)\right\} \bar{\rho}(\mathbf{q}) \bar{\rho}(-\mathbf{q}) .
$$

We define the spectral densities [19]

$$
\begin{aligned}
& \rho_{T}(\omega)=\frac{1}{N} \sum_{n}\left|\left\langle n\left|\int d \mathbf{x} T_{z z}\right| 0\right\rangle\right|^{2} \delta\left(\omega-E_{n}\right), \\
& \bar{\rho}_{T}(\omega)=\frac{1}{N} \sum_{n}\left|\left\langle n\left|\int d \mathbf{x} T_{\bar{z} \bar{z}}\right| 0\right\rangle\right|^{2} \delta\left(\omega-E_{n}\right),
\end{aligned}
$$

where $N$ is the total number of electrons, $|0\rangle$ is the ground state, the sum is taken over all excited states $|n\rangle$ in the lowest Landau level, and $E_{n}$ is the energy of the state $|n\rangle$. The two spectral densities satisfy the sum rules [19]

$$
\begin{aligned}
& \int_{0}^{\infty} \frac{d \omega}{\omega^{2}}\left[\rho_{T}(\omega)-\bar{\rho}_{T}(\omega)\right]=\frac{\bar{s}}{4}, \\
& \int_{0}^{\infty} \frac{d \omega}{\omega^{2}}\left[\rho_{T}(\omega)+\bar{\rho}_{T}(\omega)\right]=S_{4},
\end{aligned}
$$

where $\bar{s}$ is the "guiding center spin" [18], which is equal to $(\mathcal{S}-1) / 2$ on the LLL, where $\mathcal{S}$ is the shift, and $S_{4}$ is the coefficient in front of $\left(k \ell_{B}\right)^{4}$ in the static structure factor.
[1] D. C. Tsui, H. L. Stormer, and A. C. Gossard, Two-Dimensional Magnetotransport in the Extreme Quantum Limit, Phys. Rev. Lett. 48, 1559 (1982).

[2] R. B. Laughlin, Anomalous Quantum Hall Effect: An Incompressible Quantum Fluid with Fractionally Charged Excitations, Phys. Rev. Lett. 50, 1395 (1983).

[3] B. I. Halperin, Statistics of Quasiparticles and the Hierarchy of Fractional Quantized Hall States, Phys. Rev. Lett. 52, 1583 (1984).

[4] F. Wilczek, Magnetic Flux, Angular Momentum, and Statistics, Phys. Rev. Lett. 48, 1144 (1982).

[5] N. Read and D. Green, Paired states of fermions in two dimensions with breaking of parity and time-reversal symmetries and the fractional quantum Hall effect, Phys. Rev. B 61, 10267 (2000).

[6] Z. F. Ezawa, Quantum coherence and Skyrmions in a bilayer quantum Hall system, Phys. Rev. B 55, 7771 (1997).

[7] A. Gromov and D. T. Son, Bimetric Theory of Fractional Quantum Hall States, Phys. Rev. X 7, 041032 (2017).

[8] G. Moore and N. Read, Nonabelions in the fractional quantum Hall effect, Nucl. Phys. B 360, 362 (1991).

[9] C. Nayak, S. H. Simon, A. Stern, M. Freedman, and S. Das Sarma, Non-Abelian anyons and topological quantum computation, Rev. Mod. Phys. 80, 1083 (2008).

[10] S. M. Girvin, A. H. MacDonald, and P. M. Platzman, Magnetoroton theory of collective excitations in the fractional quantum Hall effect, Phys. Rev. B 33, 2481 (1986).

[11] A. Pinczuk, B. Dennis, L. Pfeiffer, and K. West, Light scattering by collective excitations in the fractional quantum Hall regime, Physica B: Condens. Matter 249-251, 40 (1998).

[12] M. Kang, A. Pinczuk, B. S. Dennis, M. A. Eriksson, L. N. Pfeiffer, and K. W. West, Inelastic Light Scattering by Gap Excitations of Fractional Quantum Hall States at $1 / 3 \leqslant v \leqslant 2 / 3$, Phys. Rev. Lett. 84, 546 (2000).
[13] I. V. Kukushkin, J. H. Smet, V. W. Scarola, V. Umansky, and K. von Klitzing, Dispersion of the excitations of fractional quantum Hall states, Science 324, 1044 (2009).

[14] P. Wiegmann, Inner Nonlinear Waves and Inelastic Light Scattering of Fractional Quantum Hall States as Evidence of the Gravitational Anomaly, Phys. Rev. Lett. 120, 086601 (2018).

[15] P. M. Platzman and S. He, Resonant Raman scattering from mobile electrons in the fractional quantum Hall regime, Phys. Rev. B 49, 13674 (1994).

[16] J. C. Collins, D. E. Soper, and G. F. Sterman, Factorization of hard processes in QCD, Adv. Ser. Direct. High Energy Phys. 5, 1 (1989).

[17] J. M. Luttinger, Quantum theory of cyclotron resonance in semiconductors: general theory, Phys. Rev. 102, 1030 (1956).

[18] F. D. M. Haldane, Geometrical Description of the Fractional Quantum Hall Effect, Phys. Rev. Lett. 107, 116801 (2011).

[19] S. Golkar, D. X. Nguyen, and D. T. Son, Spectral sum rules and magneto-roton as emergent graviton in fractional quantum Hall effect, JHEP 01 (2016) 021.

[20] S.-F. Liou, F. D. M. Haldane, K. Yang, and E. H. Rezayi, Chiral Gravitons in Fractional Quantum Hall Liquids, Phys. Rev. Lett. 123, 146801 (2019).

[21] D. T. Son, Chiral metric hydrodynamics, Kelvin circulation theorem, and the fractional quantum Hall effect, arXiv:1907.07187.

[22] L. D. Barron, Molecular Light Scattering and Optical Activity (Cambridge University Press, Cambridge, 2004).

[23] F. A. Blum, Inelastic light scattering from semiconductor plasmas in a magnetic field, Phys. Rev. B 1, 1125 (1970).

[24] D. X. Nguyen, D. T. Son, and C. Wu, Lowest Landau level stress tensor and structure factor of trial quantum Hall wave functions, arXiv:1411.3316. 
[25] Z. Liu, A. Gromov, and Z. Papić, Geometric quench and nonequilibrium dynamics of fractional quantum Hall states, Phys. Rev. B 98, 155140 (2018).

[26] T. Ruf, Phonon Raman Scattering in Semiconductors, Quantum Wells and Superlattices (Springer, Berlin, 1998).

[27] S. Golkar, D. X. Nguyen, M. M. Roberts, and D. T. Son, Higher-Spin Theory of the Magnetorotons, Phys. Rev. Lett. 117, 216403 (2016).

[28] D. X. Nguyen, S. Golkar, M. M. Roberts, and D. T. Son, Particle-hole symmetry and composite fermions in fractional quantum Hall states, Phys. Rev. B 97, 195314 (2018).

[29] K. Yang (private communication).

[30] B. Yang, Z.-X. Hu, C. H. Lee, and Z. Papić, Generalized Pseudopotentials for the Anisotropic Fractional Quantum Hall Effect, Phys. Rev. Lett. 118, 146403 (2017).

[31] Z. Liu, A. C. Balram, Z. Papić, and A. Gromov, Quench Dynamics of Collective Modes in Fractional Quantum Hall Bilayers, Phys. Rev. Lett. 126, 076604 (2021).

[32] M. Banerjee, M. Heiblum, V. Umansky, D. E. Feldman, Y. Oreg, and A. Stern, Observation of half-integer thermal Hall conductance, Nature 559, 205 (2018).

[33] B. Dutta, W. Yang, R. A. Melcer, H. K. Kundu, M. Heiblum, V. Umansky, Y. Oreg, A. Stern, and D. Mross, Novel method distinguishing between competing topological orders, arXiv:2101.01419.

[34] D. T. Son, Is the Composite Fermion a Dirac Particle? Phys. Rev. X 5, 031027 (2015).

[35] M. Levin, B. I. Halperin, and B. Rosenow, Particle-Hole Symmetry and the Pfaffian State, Phys. Rev. Lett. 99, 236806 (2007).

[36] S.-S. Lee, S. Ryu, C. Nayak, and M. P. A. Fisher, Particle-Hole Symmetry and the $v=\frac{5}{2}$ Quantum Hall State, Phys. Rev. Lett. 99, 236807 (2007).
[37] E. H. Rezayi, Landau Level Mixing and the Ground State of the $v=5 / 2$ Quantum Hall Effect, Phys. Rev. Lett. 119, 026801 (2017).

[38] B. Lian and J. Wang, Theory of the disordered $v=\frac{5}{2}$ quantum thermal Hall state: emergent symmetry and phase diagram, Phys. Rev. B 97, 165124 (2018).

[39] D. F. Mross, Y. Oreg, A. Stern, G. Margalit, and M. Heiblum, Theory of Disorder-Induced Half-Integer Thermal Hall Conductance, Phys. Rev. Lett. 121, 026801 (2018).

[40] C. Wang, A. Vishwanath, and B. I. Halperin, Topological order from disorder and the quantized Hall thermal metal: possible applications to the $v=5 / 2$ state, Phys. Rev. B 98, 045112 (2018).

[41] P.-S. Hsin, Y.-H. Lin, N. M. Paquette, and J. Wang, Effective field theory for fractional quantum Hall systems near $v=5 / 2$, Phys. Rev. Research 2, 043242 (2020).

[42] S. H. Simon, Interpretation of thermal conductance of the $v=$ 5/2 edge, Phys. Rev. B 97, 121406(R) (2018).

[43] D. E. Feldman, Comment on "Interpretation of thermal conductance of the $v=5 / 2$ edge," Phys. Rev. B 98, 167401 (2018).

[44] K. K. W. Ma and D. E. Feldman, Partial equilibration of integer and fractional edge channels in the thermal quantum Hall effect, Phys. Rev. B 99, 085309 (2019).

[45] S. H. Simon and B. Rosenow, Partial Equilibration of the AntiPfaffian Edge due to Majorana Disorder, Phys. Rev. Lett. 124, 126801 (2020).

[46] F. D. M. Haldane, E. H. Rezayi, and K. Yang, Graviton chirality and topological order in the half-filled Landau level, arXiv:2103.11019 [cond-mat.mes-hall].

[47] J. W. Haus, Nanophotonic devices, in Fundamentals and Applications of Nanophotonics (Woodhead Publishing, 2016), pp. 341-395. 